\title{
Canonical derivation of the gluon propagator in the temporal gauge
}

\author{
H. O. Girotti* and H. J. Rothe \\ Institut für Theoretische Physik der Universität Heidelberg, D-6900 Heidelberg, Germany
}

(Received 5 Septemper 1985)

\begin{abstract}
We reexamine the problem of obtaining, within the operator approach, an unambiguous expression for the longitudinal gluon propagator in the temporal gauge. A regularization procedure respecting Gauss's law and the Hermiticity of the gauge fields is proposed. We thereby obtain a definite expression for the longitudinal propagator which agrees with that proposed by Caracciolo, Curci, and Menotti.
\end{abstract}

In the past years there has been much controversy about the structure of the longitudinal part of the gluon propagator $\left[D_{L}^{j, a b}(x, y)\right]$ in the temporal gauge. ${ }^{1-5}$ The difficulty in obtaining an unambiguous expression for this propagator is connected with the problem of implementing Gauss's law as a subsidiary condition on the physical state vectors. The propagator obtained by Frenkel, ${ }^{1}$ which corresponds to the principal-value prescription, ${ }^{6,7}$

$i D_{L}^{i j, a b}(x, y)=-\frac{i}{2} \delta^{a b} \epsilon\left(x^{0}-y^{0}\right)\left(x^{0}-y^{0}\right) \frac{\partial_{x}^{i} \partial_{x}^{j}}{\nabla_{x}^{2}} \delta^{(3)}(\mathbf{x}-\mathbf{y})$,

leads to inconsistencies in $\mathrm{QCD} .^{2,3}$ The removal of these inconsistencies calls for additional terms, as was shown by Caracciolo, Curci, and Menotti, ${ }^{3}$ who arrived at the follow- ing expression:

$$
\begin{aligned}
i D_{L}^{i, a b}(x, y)=-\frac{i}{2} \delta^{a b}[\epsilon & \left(x^{0}-y^{0}\right)\left(x^{0}-y^{0}\right) \\
& \left.+\alpha\left(x^{0}+y^{0}\right)+\gamma\right] \frac{\partial_{x}^{i} \partial_{x}^{j}}{\nabla_{x}{ }^{2}} \delta^{(3)}(\mathbf{x}-\mathbf{y}),
\end{aligned}
$$

where $\gamma$ is a constant, and $\alpha$ was determined to be $\alpha= \pm 1$, based on the requirement of gauge invariance for the Wilson loop. This kind of argument does not serve to fix the value of $\alpha$ in quantum electrodynamics (QED) where also the $S$ matrix appears to be insensitive to the presence of the additional term proportional to $\left(x^{0}+y^{0}\right)$. In Ref. 4 an attempt was made to obtain (2) from first principles. By working with a dressed vacuum, Dahmen, Scholz, and Steiner ${ }^{4}$ were led to

$$
i D_{L}^{i j, a b}(x, y)=-\frac{i}{2} \delta^{a b}\left(\epsilon\left(x^{0}-y^{0}\right)\left(x^{0}-y^{0}\right)-\frac{1-\lambda}{1+\lambda} x^{0} y^{0}\left(\nabla_{x}{ }^{2}\right)^{1 / 2}+\frac{1+\lambda}{1-\lambda}\left(\nabla_{x}{ }^{2}\right)^{-1 / 2}\right) \frac{\partial_{x}^{i} \partial_{x}^{j}}{\nabla_{x}{ }^{2}} \delta^{(3)}(\mathbf{x}-\mathbf{y})
$$

where $\lambda$ is a regularization parameter. The limit $\lambda \rightarrow 1^{-}$is understood to be taken at the end of the calculations. Notice that no term proportional to $\left(x^{0}+y^{0}\right)$ is present in (3).

Within the functional approach, the situation appears to have been clarified. In Ref. 8 the propagator (2), with $\alpha= \pm 1$, appears as one among the possible choices for the longitudinal gluon propagator, while in Ref. 9 the propagator proposed by Caracciolo, Curci, and Menotti ${ }^{3}$ was unambiguously obtained by working in a fully fixed temporal gauge.

In this note we reexamine the problem of obtaining a unique expression for the longitudinal propagator within the operator approach. Since the essentials of the problem are already present at the level of the free Maxwellian field, it suffices to consider only this case.

The dynamics of the system is described by the Hamiltonian

$$
H=\int d^{3} x\left(\frac{1}{2} \Pi_{j} \Pi_{j}+\frac{1}{4} F^{i j} F^{i j}\right),
$$

together with the equal-time commutation relations

$$
\begin{aligned}
& {\left[A^{i}\left(x^{0}, \mathbf{x}\right), A^{j}\left(y^{0}, \mathbf{y}\right)\right]_{x^{0}=y^{0}}=\left[\Pi_{i}\left(x^{0}, \mathbf{x}\right), \Pi_{j}\left(y^{0}, \mathbf{y}\right)\right]_{x^{0}=y^{0}}=0,} \\
& {\left[A^{i}\left(x^{0}, \mathbf{x}\right), \Pi_{j}\left(y^{0}, \mathbf{y}\right)\right]_{x^{0}=y^{0}}=i \delta_{j}^{i} \delta^{(3)}(\mathbf{x}-\mathbf{y}),}
\end{aligned}
$$

where the $\Pi_{j}$ 's are the momenta canonically conjugate to the field potentials $A^{j}$ and $F^{i j}=\partial^{i} A^{j}-\partial^{j} A^{i}$. Since (5b) does not allow for implementing $\partial \Pi_{j}=0$ as a strong operator relation, Gauss's law is incorporated by restricting the physical states to satisfy

$$
\partial \Pi_{j}|\psi\rangle=0
$$

From Eqs. (4) and (5) it is immediately seen that the transversal $\left(A_{T}^{i}, \Pi_{i T}\right)$ and longitudinal $\left(A_{L}^{i}, \Pi_{i L}\right)$ degrees of freedom decouple and, in fact, the propagator is given as the sum of a transversal $\left[D_{T}^{i j}(x, y)\right]$ and a longitudinal part $\left[D_{L}^{i j}(x, y)\right] .^{1}$ The solution for $D_{T}^{i j}$ is, of course, well known. On the other hand, the integration of the Heisenberg equations of motion leads to the following solution for the longitudinal part of the potential: ${ }^{4}$

$$
\begin{aligned}
A_{L}^{i}\left(x^{0}, \mathbf{x}\right)=\partial_{x}^{i}[ & \left(-\nabla_{x}{ }^{2}\right)^{-3 / 4} \chi_{+}(\mathbf{x}) \\
& \left.+x^{0}\left(-\nabla_{x}^{2}\right)^{-1 / 4} \chi_{-}(\mathbf{x})\right],
\end{aligned}
$$

where $\chi_{+}$and $\chi_{-}$are time-independent Hermitian fields obeying the commutation relations

$$
\begin{aligned}
& {\left[\chi_{+}(\mathbf{x}), \chi_{+}(\mathbf{y})\right]=\left[\chi_{-}(\mathbf{x}), \chi_{-}(\mathbf{y})\right]=0,} \\
& {\left[\chi_{+}(\mathbf{x}), \chi_{-}(\mathbf{y})\right]=i \delta^{(3)}(\mathbf{x}-\mathbf{y})}
\end{aligned}
$$

In terms of these fields, condition (6) becomes

$$
\left.\left.\chi_{-}(\mathbf{x})\right|_{\psi}\right\rangle_{L}=0
$$


where we have made explicit that the subsidiary condition (6) only operates in the "longitudinal" space of state vectors.

We next pinpoint the basic difficulty encountered in computing the longitudinal propagator

$$
i D_{L}^{\psi}(x, y) \equiv{ }_{L}\left\langle\psi\left|T\left(A_{L}^{i}\left(x^{0}, \mathbf{x}\right) A_{L}^{j}\left(y^{0}, \mathbf{y}\right)\right)\right| \psi\right\rangle_{L},
$$

which according to (7) demands the evaluation of matrix elements involving products of $\chi_{+}$and $\chi_{-}$such as

$$
{ }_{L}\left\langle\psi\left|\chi_{ \pm}(\mathbf{x}) \chi_{\mp}(\mathbf{y})\right| \psi\right\rangle_{L} .
$$

The Hermiticity of $A_{L}^{i}$ requires $\chi_{+}$and $\chi_{-}$to be Hermitian fields. Now the Hilbert space supporting this Hermiticity property consists only of normalizable states, while the states annihilated by $\chi_{-}$are of infinite norm. Therefore, the operator $\chi_{-}(\mathbf{x})$ cannot be diagonalized in the space defined by (9) and, hence, the matrix elements displayed in (11) are undefined..$^{10}$

Thus, a regularization procedure is needed to ensure the compatibility of the Hermiticity of $\chi_{+}$and $\chi_{-}$with the subsidiary condition (9). This is not the case in the work of Ref. 4, where the longitudinal vacuum $|\psi\rangle_{L}$ was replaced by a normalizable dressed vacuum $\left|\Omega_{\lambda}\right\rangle$. For all values $\lambda<1$ the state $\left|\Omega_{\lambda}\right\rangle$ secures the implementation of the commutation rules (8) and preserves the Hermiticity of $\chi_{+}$and $\chi_{-}$ but violates Gauss's law (9). Although for $\lambda \rightarrow 1^{-}$the state remains normalizable and is formally annihilated by $\chi_{-}$, Gauss's law is not implemented in this limit as can be seen by computing with the regularization technique of Ref. 4, the matrix element (11):

$$
\lim _{\lambda \rightarrow 1^{-}}\left\langle\Omega_{\lambda}\left|\chi_{ \pm}(\mathbf{x}) \chi_{\mp}(\mathbf{y})\right| \Omega_{\lambda}\right\rangle= \pm \frac{1}{2} i \delta^{(3)}(\mathbf{x}-\mathbf{y}) .
$$

It is the particular structure of the matrix elements (12) which is responsible for the absence of a term proportional to $\left(x^{0}+y^{0}\right)$ in $(3)$.

To motivate the regularization scheme to be proposed in this paper, it is instructive to study the regularization procedure of Ref. 4 within the context of one-dimensional nonrelativistic quantum mechanics. From the commutation relations (8) it is clear that $\chi_{+}$and $\chi_{-}$play the role of the position $(X)$ and linear momentum operator $(P)$, respectively. Hence, the subsidiary condition (9) translates into

$$
P|p=0\rangle=0 \text {. }
$$

To construct the state analogous to $\left|\Omega_{\lambda}\right\rangle$, hereafter denoted by $\left|\omega_{\lambda}\right\rangle$, we start from the equation satisfied by $\left|\Omega_{\lambda}\right\rangle$ in field theory ${ }^{4,11}$

$$
\chi_{-}(\mathbf{x})\left|\Omega_{\lambda}\right\rangle=i \Delta(\lambda) \chi_{+}(\mathbf{x})\left|\Omega_{\lambda}\right\rangle,
$$

where $\Delta(\lambda)=(1-\lambda) /(1+\lambda)$. The corresponding equation in the nonrelativistic case then reads

$$
P\left|\omega_{\lambda}\right\rangle=i \Delta(\lambda) X\left|\omega_{\lambda}\right\rangle,
$$

whose normalized solution in the position representation $\left(\omega_{\lambda}(x)=\left\langle x \mid \omega_{\lambda}\right\rangle\right)$ is given by

$$
\omega_{\lambda}(x)=\left(\frac{\Delta(\lambda)}{\pi}\right)^{1 / 4} \exp \left[-\frac{1}{2} \Delta(\lambda) x^{2}\right] .
$$

For $\Delta(\lambda)>0(\lambda<1)$, Eq. (16) describes a wave packet with a momentum distribution $\exp \left[-p^{2} / 2 \Delta(\lambda)\right]$. Although $\left|\omega_{\lambda}\right\rangle$ is annihilated by $P$ in the limit $\Delta \rightarrow 0^{+}\left(\lambda \rightarrow 1^{-}\right)$, one finds that

$$
\begin{aligned}
& \lim _{\Delta(\lambda) \rightarrow 0^{+}}\left\langle\omega_{\lambda}|X P| \omega_{\lambda}\right\rangle=\frac{1}{2} i, \\
& \lim _{\Delta(\lambda) \rightarrow 0^{+}}\left\langle\omega_{\lambda}|P X| \omega_{\lambda}\right\rangle=-\frac{1}{2} i,
\end{aligned}
$$

which shows that (13) has not even been implemented in the limit $\Delta(\lambda) \rightarrow 0^{+}$. In summary, the replacement of the eigenstate $|p=0\rangle$ by a normalized wave packet centered around $p=0$ is not an acceptable regularization method for the problem under analysis.

We shall next use the nonrelativistic quantum-mechanical formulation of the problem to propose an alternative regularization procedure which secures the implementation of (13) at all stages of the calculation, while keeping the Hermiticity of $X$ and $P$. To this effect we begin by restricting the eigenvalues of $X$ to the finite domain $-\Lambda \leqslant x$ $\leqslant+\Lambda$, the limit $\Lambda \rightarrow \infty$ being taken at the end of the calculations. Then, the requirement of $P$ to be Hermitian implies that the wave functions $\phi(x)$ satisfy periodic or quasiperiodic boundary conditions, namely, $\phi(\Lambda) / \phi(-\Lambda)$ $=\exp (i \theta)$, where $\theta$ is an arbitrary constant phase. In order for the discrete spectrum of $P$, labeled by the integer $n$, to contain the eigenvalue $p=0$, as required by (13), we restrict the wave functions to be strictly periodic. Thus our regularization scheme amounts to compactifying the space. The computation of the diagonal matrix elements of the operators $X P$ and $P X$ between zero-momentum $(n=0)$ eigenstates now yields

$$
\begin{aligned}
& \langle n=0|X P| n=0\rangle=0, \\
& \langle n=0|P X| n=0\rangle=-i .
\end{aligned}
$$

The striking difference between (17) and (18) should be noticed. We remark that (18a) and (18b) are both consistent with the subsidiary condition (13); indeed, since $P$ is not Hermitian with regard to the nonperiodic state $X|n=0\rangle$, there are surface terms contributing to (18b) when $P$ acts to the left. These surface terms precisely ensure the validity of the commutation rule $[X, P]=i$. For a "subsidiary condition" like (13) one can avoid the explicit computation of such surface terms by letting all operators act only to the right.

At this point a digression is in order. Within the regularization approach proposed in this paper it is immediately seen that the erroneous conclusion arrived at in Ref. 12 amounts to neglecting the above-mentioned surface terms. The fact that the Hermitian character of the Gauss-law operator was not properly handled in Ref. 12 has been pointed out by several authors. ${ }^{13}$ However, no prescription was given in the works of Ref. 13 for computing the longitudinal gauge-field propagator within the operator approach. To provide such a prescription has been the purpose of this paper.

To exemplify how different regularization techniques lead to different results we compute the expression analogous to (10) for a free unit-mass particle moving in a one-dimensional space. In this case the Heisenberg-picture operators $X(t)$ and $P(t)$ are given in terms of the corresponding Schrödinger-picture operators $(X, P)$ by the relations $P(t)$ $=P, X(t)=X+t P$. By using the regularization scheme in- 
troduced in this work one finds

$$
\begin{aligned}
\langle n=0| T & \left(X(t) X\left(t^{\prime}\right)\right)|n=0\rangle \\
& =-\frac{1}{2} i\left[\epsilon\left(t-t^{\prime}\right)\left(t-t^{\prime}\right)+\left(t+t^{\prime}\right)\right]+\frac{1}{3} \Lambda^{2},
\end{aligned}
$$

which already exhibits the presence of a term proportional to $\left(t+t^{\prime}\right)$. On the other hand, the nonrelativistic version of the regularization procedure of Ref. 4 leads to

$$
\begin{aligned}
\left\langle\omega_{\lambda}\left|T\left(X(t) X\left(t^{\prime}\right)\right)\right| \omega_{\lambda}\right\rangle= & -\frac{1}{2} i \epsilon\left(t-t^{\prime}\right)\left(t-t^{\prime}\right) \\
& +\frac{\Delta(\lambda)}{2} t t^{\prime}+\frac{1}{2 \Delta(\lambda)} .
\end{aligned}
$$

where no term proportional to $\left(t+t^{\prime}\right)$ shows up. Furthermore, in the work of Frenkel ${ }^{1}$ time-ordered products were computed by using the identity

$T\left(X(t) X\left(t^{\prime}\right)\right)=\frac{1}{2} \epsilon\left(t-t^{\prime}\right)\left[X(t), X\left(t^{\prime}\right)\right]+\frac{1}{2}\left\{X(t), X\left(t^{\prime}\right)\right\}$.

When the right-hand side of (21) is evaluated using our regularization procedure one comes back to (19). Frenkel, ${ }^{1}$ however, ignored the contribution of the anticommutator in (21), which amounts to neglecting the contribution of the surface terms, and, hence, he is left with the principal-value prescription

$\left\langle p=0\left|T\left(X(t) X\left(t^{\prime}\right)\right)\right| p=0\right\rangle=-\frac{1}{2} i \epsilon\left(t-t^{\prime}\right)\left(t-t^{\prime}\right)$.

We now come back to the field-theory case. The Hamiltonian (4) splits into a transverse $\left(H_{T}\right)$ and a longitudinal $\left(H_{L}\right)$ part which are, respectively, given by

$$
\begin{aligned}
& H_{T}=\int d^{3} x\left(\frac{1}{2} \Pi_{j T} \Pi_{j T}+\frac{1}{4} F^{v} F^{U}\right), \\
& H_{L}=\int d^{3} x \frac{1}{2} \Pi_{J L} \Pi_{J L} .
\end{aligned}
$$

Hence, as far as the longitudinal degrees of freedom are concerned (which we recall decouple completely from the transverse ones), the situation is quite analogous to that of a free particle in nonrelativistic quantum mechanics. Furthermore, our regularization scheme can be formally extended to the field-theory case by working in a representation where $\chi_{+}(\mathbf{x})$ is diagonal. To avoid the problem of evaluating "surface terms" in field space, we compute the longitudinal propagator by letting all operators act to the right in the corresponding maxtrix elements. In this way we find

$$
\begin{aligned}
& { }_{L}\left\langle\psi\left|T\left(A_{L}^{l}\left(x^{0}, \mathbf{x}\right) A_{L}^{j}\left(y^{0}, \mathbf{y}\right)\right)\right| \psi\right\rangle_{L} \\
& =-\frac{1}{2} i\left[\epsilon\left(x^{0}-y^{0}\right)\left(x^{0}-y^{0}\right)+\left(x^{0}+y^{0}\right)\right] \frac{\partial_{x}^{l} \partial_{x}^{j}}{\nabla_{x}^{2}} \delta^{(3)}(\mathbf{x}-\mathbf{y}),
\end{aligned}
$$

where we have ignored a time-independent term which does not contribute to gauge-invariant quantities. Except for a trivial Kroenecker $\delta$ function in the color indices, the same expression holds for the longitudinal gluon propagator in QCD. Expression (25) is in agreement with (2) for $\alpha=+1$. Nevertheless, and in contradistinction to Ref. 3, we have arrived at a definite form for the longitudinal gluon propagator without invoking any consistency argument for the Wilson loop.

One of us (H.O.G.) is indebted to the Institut für Theoretische Physik der Universität Heidelberg for the kind hospitality extended to him during the realization of this work, and would like also to thank the Conselho Nacional de Desenvolvimento Cientifico e Tecnologico (CNPq), Brazil, for financial support.
'On leave of absence from the instituto de Fisica, Universidade do Rio Grande do Sul, Brazil.

1J. Frenkel, Phys. Lett. 85B, 63 (1979); see also, J. Frenkel and J. C. Taylor, Nucl. Phys. B109, 439 (1976).

${ }^{2}$ V. F. Müller and W. Rühl, Ann. Phys. (N.Y.) 133, 240 (1981).

${ }^{3}$ S. Caracciolo, G. Curci, and P. Menotti, Phys. Lett. 113B, 311 (1982).

${ }^{4}$ H. D. Dahmen, B. Scholz, and F. Steiner, Phys. Lett. 117B, 339 (1982).

${ }^{5}$ B. Scholz and F. Steiner, DESY Report No. 83-055, 1983 (unpublished).

${ }^{6}$ W. Kummer, Acta Phys. Austriaca 41, 315 (1975).

${ }^{7}$ W. Konetschny and W. Kummer, Nucl. Phys. B100, 106 (1975).

${ }^{8}$ J. P. Leroy, J. Micheli, and G. C. Rossi, Nucl. Phys. B232, 511 (1984).
${ }^{9}$ H. O. Girotti and K. D. Rothe, Z. Phys. C 27, 559 (1985).

${ }^{10} \mathrm{This}$ can easily be seen in the representation where the operator $\chi_{+}(\mathbf{x})$ is diagonal and $\chi_{-}(\mathbf{x})$ is realized as $-i \delta / \delta x_{+}(\mathbf{x})$.

${ }^{11} R$. Utiyama, T. Imamura, S. Sunakawa, and T. Dodo, Prog. Theor. Phys. 6, 587 (1951); see also, J. M. Jauch and F. Rohrlich, The Theory of Photons and Electrons, 2nd ed. (Springer, New York, 1980).

${ }^{12}$ Y. Kakudo, Y. Taguchi, A. Tanaka, and K. Yamamoto, Phys. Rev. D 27, 1954 (1983).

${ }^{13}$ M. Hossein Partovi, Phys. Rev. D 29, 2993 (1984); B. F. Hatfield, ibid. 29, 2995 (1984); G. C. Rossi and M. Testa, ibid. 29, 2997 (1984); I. Bialynicki-Birula and P. Kurzepa, ibid. 29, 3000 (1984). 